\title{
A GRAPHICAL APPROACH TO TESTING \\ REAL-TIME EMBEDDED DEVICES
}

\author{
A Thesis \\ presented to \\ the Faculty of California Polytechnic State University, \\ San Luis Obispo
}

\author{
In Partial Fulfillment \\ of the Requirements for the Degree \\ Master of Science in Computer Science
}

by

Steven Michael Day

June 2009 
(C) 2009

Steven Michael Day

ALL RIGHTS RESERVED 


\section{COMMITTEE MEMBERSHIP}

TITLE:

AUTHOR:

DATE SUBMITTED:

COMMITTEE CHAIR:

COMMITTEE MEMBER: Dr. Franz Kurfess

COMMITTEE MEMBER: $\quad$ Dr. Clark Turner

Dr. Tim Kearns
A Graphical Approach to Testing Real-Time Embedded Devices

Steven Michael Day

June 2009 


\begin{abstract}
A Graphical Approach to Testing Real-Time Embedded Devices

by
\end{abstract}

Steven Michael Day

Software Testing is both a vital and expensive part of the software development lifecycle. Improving the testing process has the potential for large returns. Current testing methodologies used to test real-time embedded devices are examined and the weaknesses in them are exposed. This leads to the introduction of a new graphical testing methodology based on flowcharts. The new approach is both a visual test creation program and an automated execution engine that together frame a new way of testing. The new methodology incorporates flow-based diagrams, visual layouts, and simple execution rules to improve upon traditional testing approaches. The new methodology is evaluated against other methodologies and is shown to provide significant improvements in the area of software testing. 


\section{Contents}

List of Figures ............................................................................................................................................ vii

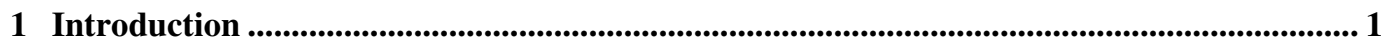

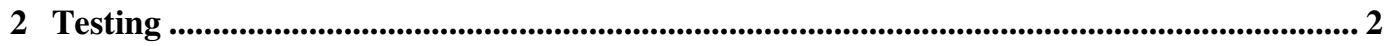

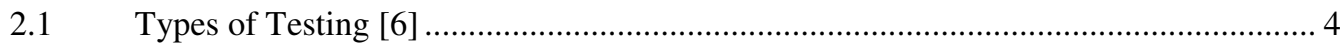

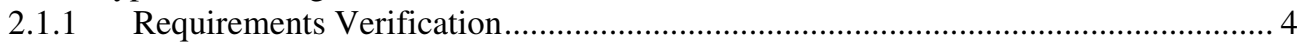

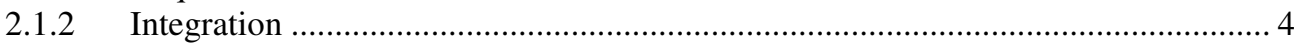

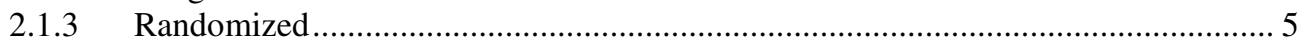

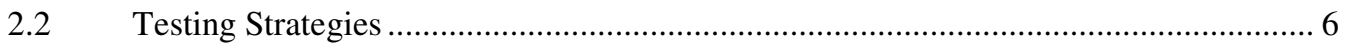

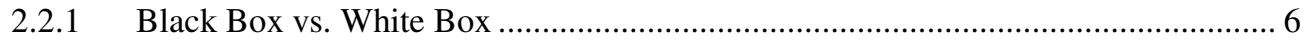

2.2.2 Breadboards vs. Emulators ............................................................................. 9

3 Embedded System Testing Approaches............................................................................................ 12

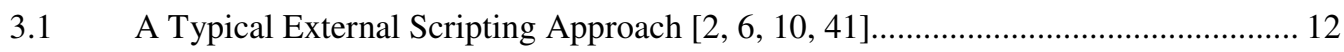

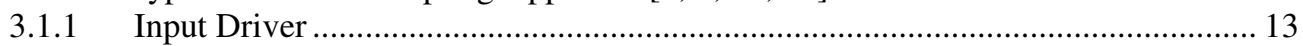

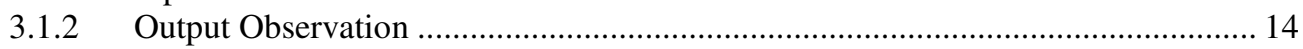

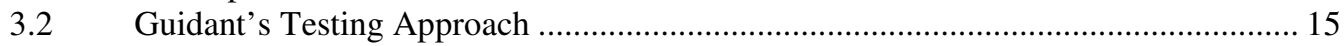

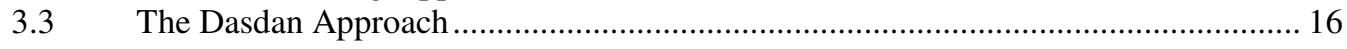

4 Graphical Flowchart Approach ............................................................................ 18

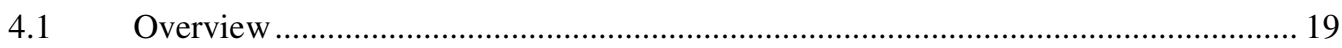

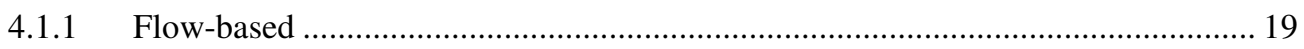

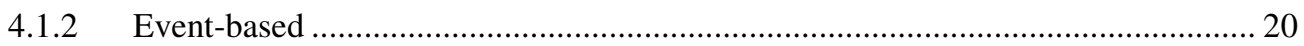

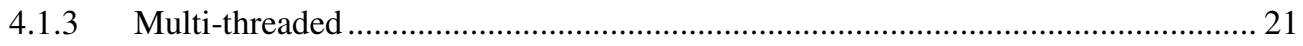

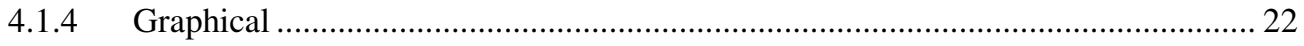

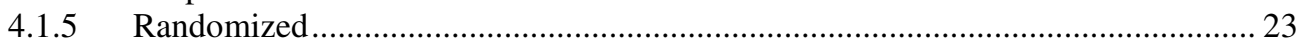

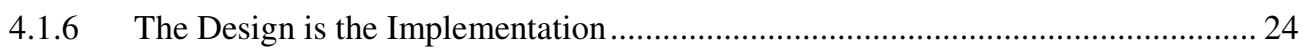

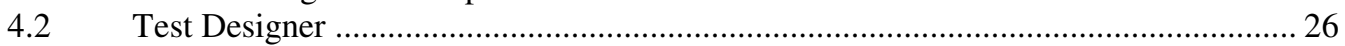

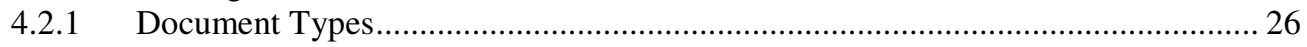

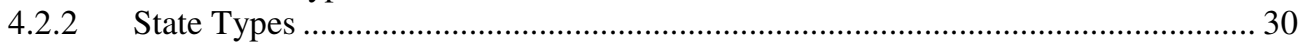

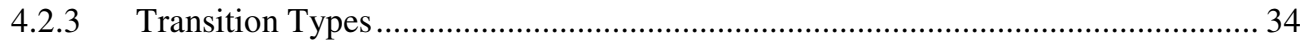

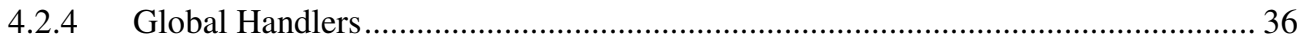

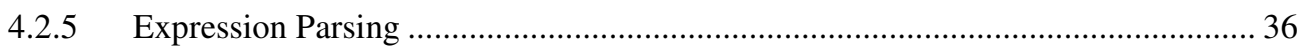

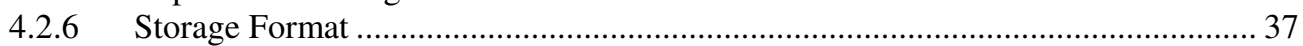

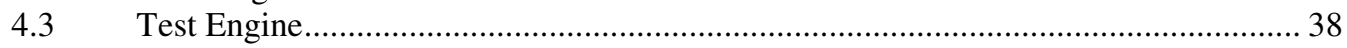

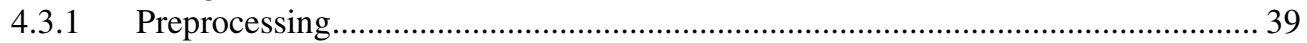

4.3.2 Flow Processing and Event Processing …………............................................... 39

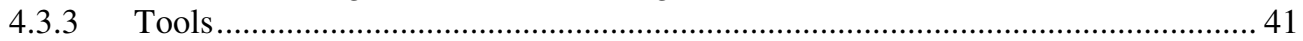

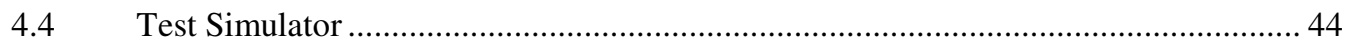

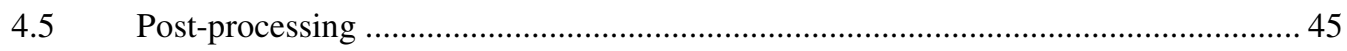




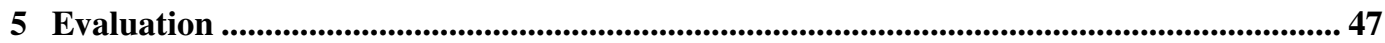

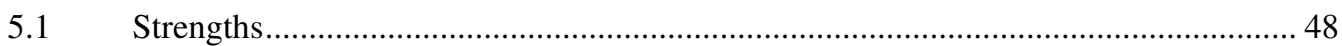

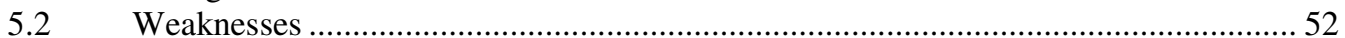

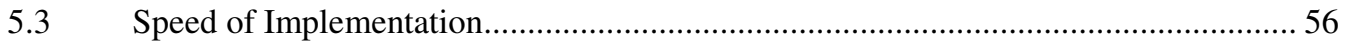

6 Future Work ................................................................................................................................................ 58

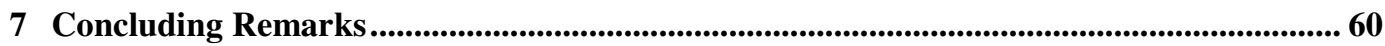

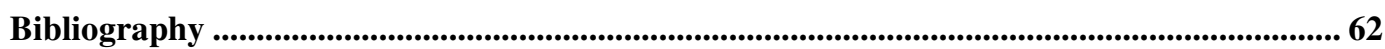




\section{List of Figures}

4.1 GiFT System Overview .................................................................19

4.2 Event-based processing. The execution will proceed from the initial state to the success state when the event Sig.Charging_Completed is

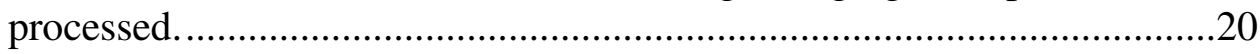

4.3 Multiple flows simulate a multi-threaded application ................................21

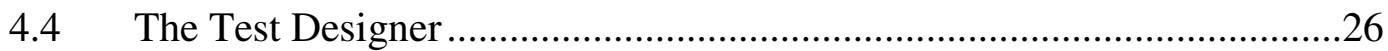

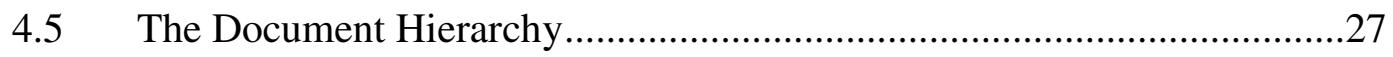

4.6 Ordinary state ....................................................................................

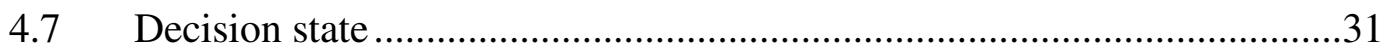

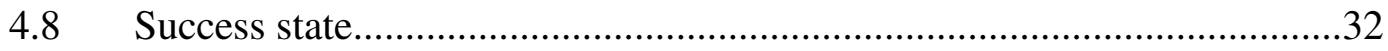

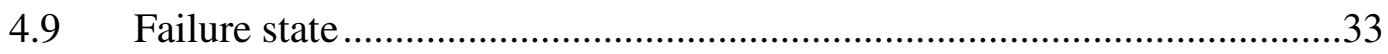

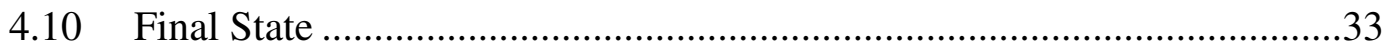

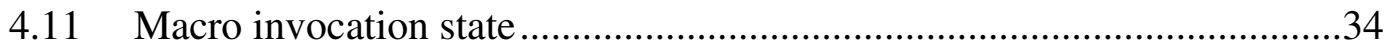

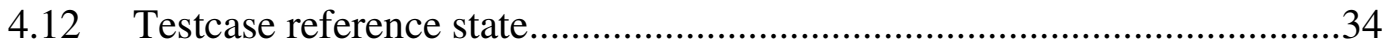

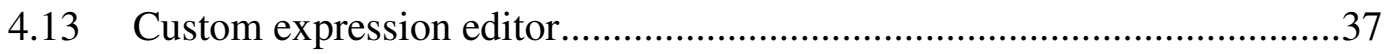

5.1 GiFT vs. Typical Approach Comparison................................................57 


\section{Chapter 1}

\section{Introduction}

As a society, we are becoming increasingly dependent on embedded devices to perform safety-critical operations. These embedded devices may be found in such applications as automobiles, avionics, nuclear power plants, and medical devices. Due to their critical nature, we place an utmost importance on the testing of these products. While being completely necessary, the large effort spent on testing is quite expensive [6]. If the efficiency of the testing activity can be improved while maintaining or improving its effectiveness, there is potential for enormous gains.

In this thesis, various testing activities will be examined with an emphasis on the common approaches used by embedded systems companies. After the overview, an entirely new paradigm for testing embedded systems will be detailed. The deficiencies of the common approaches will be identified and analyzed in the context of the new paradigm. The new paradigm will be evaluated against the other approaches to illustrate its strengths and weaknesses. In the end, it will be shown that this new paradigm offers significant improvements over the other approaches while preserving the effectiveness of the testing activity. 


\section{Chapter 2}

\section{Testing}

Software testing is one of the most expensive phases of the software development lifecycle. The cost is particularly high when it concerns embedded systems [14]. Included in the cost breakdown is the manpower to design, execute, and verify the tests as well as the delay in time-to-market for a software product. Despite the high cost of software testing, it is performed for two reasons:

\section{Regulation}

Many embedded systems are safety-critical in nature. For these applications, there is typically a governing body that regulates the testing and manufacture of the products. For example, medical device companies are regulated by the Food and Drug Administration (FDA). The FDA has guidelines and policies that drive much of the software development process within a medical device company. Part of these guidelines include the implementation and review of software testing. Tests must be designed to cover each of the software requirements and then they must be reviewed, implemented, executed, and the results verified [42, 43]. 
Without an adequate software testing strategy, the FDA could revoke the ability for a medical device company to do business. Recent history with the medical device manufacturer Guidant has shown that the FDA will take such drastic measures when they feel a company is not performing to standards [3]. Oversight is a major motivation for embedded systems companies to maintain a thorough software testing strategy.

\section{Product Quality}

Aside from the threat of having business halted by a governing body, there is a strong motivating factor in the free marketplace to produce a quality product. In the free market, it is in the company's best interest to produce items at a higher quality and lower cost than its competitors. Software testing plays a major role towards this goal.

Recently, there have been product recalls among two major embedded systems companies $[1,31]$. Product recalls can prove disastrous for increasing business and growth. One of the ways to reduce such recalls is to release a product free of major defects. The traditional way of reducing major defects in software products is through software testing [29]. 


\subsection{Types of Testing [6]}

Software testing is a broad topic. There are many different types of software testing that occur at various stages of the software development lifecycle. The following is an overview of the various types of testing addressed in this paper:

\subsubsection{Requirements Verification}

Requirements verification is a special type of testing activity. Requirements verification can be summed up by the phrase, "Did we build what we said we were going to build?"

Requirements verification is an activity that tests the product against the product's requirements to ensure they match. There are many different approaches that may be used to this end, but the activity remains the same - to verify that the product behaves exactly as described in the requirements [11].

\subsubsection{Integration}

Integration testing focuses on testing a unit while running within a system as well as the effects of the system on a particular unit. Integration testing is a very complex, yet important activity [6]. There are many potential issues that could be identified by integration testing but would never be uncovered by unit testing alone. An example of one such issue would be a conversion method. Suppose method CalculateMPH(float time, float distanceInFeet) works well in a unit test. Suppose method ConvertToMiles(float distInFeet) also works well in a unit test. The interaction between the two is what is tested with integration testing. Suppose this call is made: CalculateMPH(60.0,ConvertToMiles(5280.0)). The problem 
here is that CalculateMPH is expecting an input in feet, but receives an input in miles. This problem isn't apparent until the interaction between the two is tested.

Integration testing is very important, but it is sometimes difficult to identify all of the interactions that are important to test [37]. This makes it difficult for anyone except an entire system expert to design such tests. The expert must understand the implementation of the system and the impact different units within the system may have on each other.

\subsubsection{Randomized}

The theory behind randomized testing is that even an expert test designer may miss designing a test for an important interaction. To help eliminate this gap, a randomized test may be constructed such that it varies the test execution each time it is run. The randomization may vary the setup of the environment before the test begins, vary different inputs, and vary different code branches of execution. As the test runs, the results are compared with expected results for the given input values and setup environment. If the actual results don't match expected results, the test is flagged as a failure.

Pseudo-randomness can also be introduced to aid in the targeting of specific, known trouble areas within the code. For example, many test failures occur at the edges of a boundary because of erroneous boundary conditions [19, 21]. In this regard, the pseudo-randomness can be distributed in such a way that the boundary conditions are tested more often that the middle of a distribution. 
One important practical consideration for randomized testing involves storing the generated values that were used in each test. Without this information, repeating the condition for debugging would prove to be quite difficult.

\subsection{Testing Strategies}

While defining the fundamental testing approach for an application, there are high-level testing strategies that must be considered. Here are two of the foremost strategic decisions that must be given consideration:

\subsubsection{Black Box vs. White Box}

Black box and white box are two different approaches to software testing. Black box is a term used to describe testing without any knowledge of the internals of the system under test. White box, also sometimes referred to as clear box, is a term used to describe testing that makes use of internal knowledge about a system's construction [32].

Black box testing is best described as testing a system by only giving it inputs and observing its outputs. Nothing about the system can be inspected aside from the outputs and nothing about the system can be altered aside from the inputs. This type of testing is usually the least complicated, but it also makes for difficult problem-solving when an error condition occurs.

Black box testing is beneficial because of its simplicity. This testing approach utilizes the same interface to the system as will be used post-production. There is no additional instrumentation, apparatus, or external device development and cost

associated with black box testing. The testing harness is easily designed because it 
utilizes a very stable, well-known interface instead of a custom, testing-only interface.

Although the simplicity of black box testing can be a benefit, it can also be a detriment. Because very little instrumentation is available with black box testing, it is difficult to diagnose errors. Another drawback of black box testing is the inability to thoroughly set up a testing scenario. For instance, if a timer rollover is to be tested, it would be beneficial to set the timer to a point right before it expires before starting the test. Unfortunately, black box testing does not allow for such modifications and requires the test to wait for the timer to count until rollover time. This has the potential of adding unnecessary time delays to the test.

White box testing is best described as testing a system with a clear view of the internal structure of the system. With white box testing, the inputs and outputs may be used for testing but there is a wide array of other options. In some cases, the inputs and outputs may be completely ignored because instrumentation may monitor and alter the internals of the system to set up and assess the successfulness of a test [26].

White box testing is beneficial because of its flexibility and ability to monitor specific conditions that cannot be isolated with black box testing. With black box testing, a test may have to wait for a timer to expire or some long series of events to occur before executing the test. With white box testing, the internals of the system may be modified to simulate the situation without the added time of waiting for the condition to occur naturally. White box testing also has the ability to be much more specific in the observations it makes. At a black box testing 
perspective, the correct output causes success, but with white box testing we can ensure that the correct values are set internally in many places and have a much clearer picture of the state of the entire system at any given point in time. This helps a great deal with the debugging of a failed test [12].

There are also disadvantages to white box testing. While the ability to monitor the software is beneficial, it is also more expensive and time-consuming to develop a testing harness to allow such invasive behavior. In addition, white box testing may give too much control and the test may stop watching the state of the system before the output is really achieved. With black box testing, it is necessary to view the output after it comes down the entire pipeline; with white box testing, we may assume our test passes because the result coming out of a single function is correct, but it may be the case that the correct result is erroneously modified on its way out of the pipeline. Another disadvantage is that errors observed in a test may be an actual error in the system, but it is possible that it may be an artifact of the testing apparatus, especially for real-time systems. Errors in testing apparatus are common in real-time system testing due to the tight timing constraints placed on the system. If the test apparatus and the system under test become out of sync, false-positive errors will likely occur that cause an error result but do not necessarily indicate a fault with the device under test. These tight timing constraints are only a problem when using white box testing due to the need to keep the test apparatus in sync with the device under test for every operation it performs. 
There are distinct advantages and disadvantages to both black box and white box testing. To get the most benefit out of testing, the approach may be a blend of both types. The trade-offs of both white and black box testing must be considered thoroughly before deciding on which approach to use for each type of testing scenario.

\subsubsection{Breadboards vs. Emulators}

Embedded systems are composed of both hardware and software [30]. To execute the software for an embedded system, the hardware must be modeled. There are two main approaches for modeling an embedded system's hardware for testing purposes. The first approach is to build the actual hardware on a test bench. This is called breadboarding. The second approach is to build a software emulator that emulates the entire device [5].

Breadboarding is the equivalent of building the actual embedded device hardware, but without the cost of doing so for the actual application. For instance, if an embedded device must be the size of a deck of playing cards and encased in a titanium shell, there is significant cost in manufacturing on such a small scale with expensive materials. If, however, the hardware was laid out onto a one foot by two foot electronics board, the circuitry remains the same but there is no need for the cost to manufacture on a small scale. In addition, any expensive casing materials, supports, etc. are removed from the equation. The result is a hardware replica of the actual device to be built. This hardware replica may also sometimes include additional instrumentation that will not be included in the final hardware manufacturing build [24]. 
The advantages of breadboarding are many. The main advantage to breadboarding is that hardware and software interactions can be tested under actual hardware conditions. It has been recognized that the boundary between hardware and software is poorly understood [38]. By using a breadboard and testing both the hardware and software systems together as a whole, the boundary between the systems is thoroughly tested as well. In addition, the hardware and software platform runs at real-time, just like the final embedded device will eventually do. Due to these factors, it is more likely that problems pertaining to hardware and software interactions will be recognized on a hardware replica.

Breadboarding includes some disadvantages - mainly related to cost. If there are many materials involved and a large number of testing stations to be built, the cost may be high. Any changes to the hardware layout are usually expensive to change because it may involve creating a new breadboard layout and replacing or updating all of the existing breadboards. Some of these costs may be avoided with on-board swappable hardware chips but there still exists a cost, especially if the number of breadboards is large.

Another approach to the testing of embedded systems is to create a software emulator that emulates the hardware part of the system. This approach is exhibited with simple emulators, such as the emulators that run on PCs to emulate popular videogame consoles. The idea here is the same, except that the emulator is emulating custom, real-time hardware instead of a well-known and recognized platform. 
The advantages of emulators include the cost effectiveness when there are many test benches to be built. The cost to build an emulator is static, and once built it can be reproduced any number of times. This is contrasted with the cost to build breadboards. Each deployment of a breadboard requires additional cost. In addition, changes and updates to the emulator are also easily distributed, unlike an update to a breadboard.

While emulators may be less expensive than breadboards over a large number of installations, there is still a high development cost. Not only is the initial development expensive, but there also exists a need to verify accuracy that the emulator is properly emulating the hardware that will be built [17]. With breadboards, the hardware is virtually identical, except for scale and additional diagnostic equipment. With an emulator, there is a painstaking verification effort that must be completed on the emulator itself before it can be used for its intent to verify software. Because the emulator is also software, there may be hardware issues lurking that remain undiscovered. Likewise, it is necessary to ensure that the emulator runs at real-time so that it doesn't obscure any problems with realtime actions that may be present.

Both breadboarding and emulating hardware are viable options for testing a real-time, embedded system. There are trade-offs with each approach that must be considered before implementing a testing strategy. 


\section{Chapter 3}

\section{Embedded System Testing Approaches}

While evaluating any new paradigm, it is useful to survey existing paradigms

for comparison. The new paradigm presented in this paper applies specifically to the testing of embedded devices from an external standpoint on a physical hardware platform. Because of this focus, the approaches used for comparison will be limited in the same fashion.

This section will describe a typical external scripting testing approach for embedded systems, the verification testing paradigm in use at one embedded systems company, and a paradigm proposed in an academic paper.

\subsection{A Typical External Scripting Approach [2, 6, 10, 41]}

Given the limitations of testing a non-emulated embedded platform from an external viewpoint, there are few limited choices in the approach. The general strategy for testing an embedded system using a breadboard is a simple inputoutput method. The embedded device is set into some starting state, inputs are driven into the device, and the state of the device is observed. To accomplish this 
approach, there must be a method for driving inputs and a method for observing the state of the device.

\subsubsection{Input Driver}

Regardless of white or black box testing approach, the decision to use a breadboard for testing causes a need to generate inputs that drive the system. One possible implementation of a test driver is a simple $\mathrm{C}++$ program. This program is usually started with a skeleton to set the breadboard into a known state, trigger the observation of outputs, perform testing steps, trigger the saving of the outputs to a file, and stop any activity occurring on the device by setting it into a resting state. To determine test success or failure, the output file must be analyzed and compared to expected output.

The workflow for a verification test engineer begins with the test template that outlines the initialization and cleanup needed for each test. The verification engineer then adds routines to the template, utilizing any pre-defined custom library calls. The last part of the test design includes laying out the criteria against which the results will be measured to determine success or failure of the test. The engineer then attempts to run the test on a test bench. At the completion of the test, there are log files and outputs from the device that are measured before a success or failure result is reported. If the test does not pass successfully, it is reexamined to determine if there is an error with the test code or if the test has uncovered an actual error with the device under test. 


\subsubsection{Output Observation}

To observe the output of the breadboard during a test, there are a number of necessary tools. These tools are mostly windows into the internals of the system that allow viewing the state of the system. These tools include the logic analyzer, digital interface, and code coverage recorder.

The logic analyzer is a common tool in the embedded system industry [17]. It is a standard tool that watches values in the hardware and triggers the recording of updates to a file. The recording is customizable such that only the values of interest may be recorded. The recording may be set up to record immediately or to record only after a certain event occurs. The resulting file is called a trace file. It is a chronological record of the events that happened within the area of firmware that was being monitored.

The digital interface allows direct viewing of data on the breadboard. The digital interface is similar to the logic analyzer in that it watches the firmware. The difference is that instead of logging actions to a file, the digital interface reports specific events back to a piece of monitoring software. This software is usually a $\mathrm{C}++$ program that is running to test the device. When the events are reported back to the software, the software may make decisions based upon the occurrence of an event.

The code coverage recorder has its own interface to the device that triggers the recording of information into a file when certain areas of the code under test are executed. After running all tests, the aggregation of all of these files may be analyzed to ensure that every piece of the code under test was executed. 


\subsection{Guidant's Testing Approach}

In a Doctoral Thesis titled, “A Requirement Verification Framework for Realtime Embedded Systems" [45], author Feng Zhu details the methodology used for verification of embedded device software at a medical device company called Guidant. His thesis covers their previous method of testing as well as the improvement he made to the process by introducing standard templates.

At Guidant Corporation, verification testing was done by writing $\mathrm{C}++$ code. Each test case was developed as a separate $\mathrm{C}++$ program and executed independently of all other test cases. The $\mathrm{C}++$ program contained all of the necessary startup, test setup, and cleanup procedures. In addition, the main part of the test contained implementation that is specific to testing the given requirement.

In his thesis [45], Feng Zhu successfully transforms the verification effort from unnecessary repetition into heavy re-use. Feng Zhu identified patterns among the entire suite of tests used at Guidant. From his pattern identification, he was able to produce eight templates that cover approximately $95 \%$ of all tests that need to be written. Armed with the library of templates, the test engineer may more quickly develop a suite of tests. The engineer is no longer required to reimplement functionality that is common to many tests of a single type.

Aside from the improvement made to the process by adding standard templates, the testing methodology still requires re-implementation of the setup and cleanup code within each test, the single test per executable, and the low-level knowledge of the test system required by the test engineer to implement a test. 
This approach offers opportunities for improvement with the new methodology that will be examined later.

\subsection{The Dasdan Approach}

In the paper, “An Interactive Validation Methodology for Embedded Systems" [13], the authors describe an approach for ensuring that timing constraints are met by the system. Their approach heavily utilizes graphs and simulation to determine the outcome of the test. They propose moving testing to higher levels of abstraction and introducing it early in the design cycle.

The approach proposed by the authors of the paper begins by abstracting the testing away from the actual implementation. The testing process relies on graphs and simulation to estimate best-case and worst-case timing scenarios. Their approach is to run the tests very early in the design cycle and revisit the design if the test results are unsatisfactory.

While this approach makes a very good point to run tests early in the design cycle, it has the pitfall that it relies mainly upon simulation to determine results of time-based tests. As stated previously in this paper, simulation has both strengths and weaknesses. One of the weaknesses of simulation is the inability to fully capture hardware and software interactions that may negatively impact timing constraints. It is important to take actual measurements from an embedded device to ensure the timing constraints are met. Due to the heavy use of simulation emphasized by the authors of "An Interactive Validation Methodology for Embedded Systems," it is a tool that would possibly be considered during design 
time, but it is not an approach that would be considered for the final device firmware verification of a safety-critical embedded application. 


\section{Chapter 4}

\section{Graphical Flowchart Approach}

To address the pitfalls of the various testing approaches, a new paradigm has been developed. It is called GiFT: Graphical interface Flowchart Tool.

The approaches detailed in the previous section and GiFT share many similarities. In fact, the testing apparatus and overall testing strategy need not change when evaluating GiFT against the typical testing approach. The real focus of the new paradigm is on the way the end-user of the test system develops test cases, executes the tests, and interprets the results.

GiFT is a test design and execution tool comprised of a flowchart-based graphical interface and an event-based processing engine. Tests are designed in the test designer application on a user's workstation before being sent to the test engine for execution. The test designs are immediately executable without the need for any additional implementation. The test engine executes the test and determines the result. 


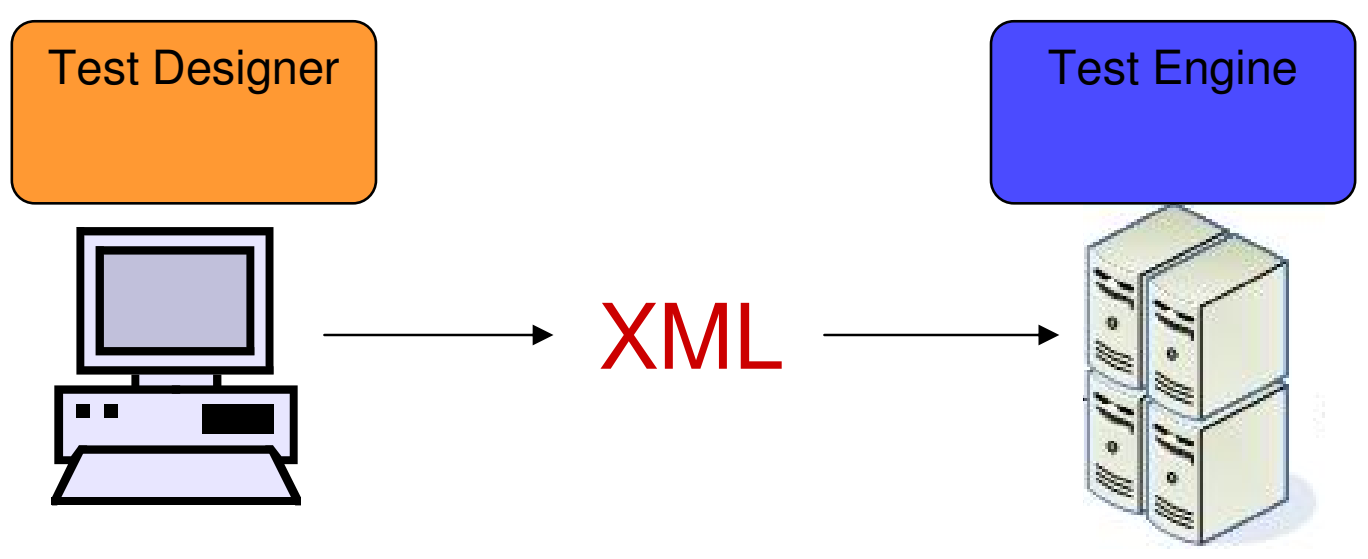

Figure 4.1: GiFT System Overview

The rest of this chapter describes the new paradigm in detail. The following chapter addresses the strengths, weaknesses, and overall evaluation of GiFT.

\subsection{Overview}

\subsubsection{Flow-based}

GiFT is based upon flowcharts. The basic design dictates that execution begins at a specified entry point, takes transitions to states based on the satisfaction of certain conditions, and ends at a terminal state that denotes the result of the test.

Because it uses flowcharts as its foundation, GiFT is a pure representation of inputs and outputs with actions that may be taken after each input. The verification test engineer specifies the test using graphical building blocks and can easily understand the connection among all the states in the test. At each state, the test author may specify certain actions to take place, such as writing a value to a variable. Finally, the execution path must end at either a success or failure state to indicate the result of the test. If the test continues to execute without reaching a 
success or failure state, a test case timer will hit its limit and stop the test. In the case of the test case timer, the test author may specify that either success or failure is reported when the timer expires.

\subsubsection{Event-based}

GiFT operates on events. Only when events occur does any action take place. The system is reactive in nature. After setting up the test scenario, the test begins in the initial state. There must be exactly one transition from the initial state to another state. If there is a condition specified on the transition out of the initial state, the test will wait for that condition to be met before transitioning to the next specified state. If there is no condition, the transition takes place immediately and the actions in the state are executed. After execution, the next transition is evaluated. If there is a condition, execution will wait until the condition is met. If not, immediate transition to the next state takes place. This will continue until the execution reaches a terminal state and the test completes or until the test case timer expires and stops the test.

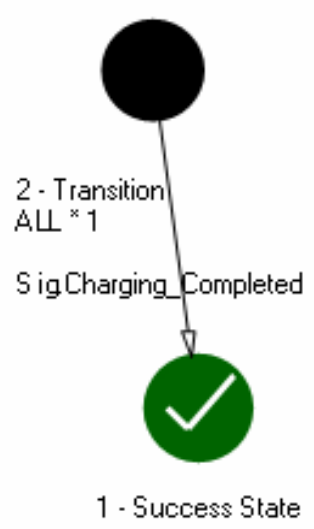

Figure 4.2: Event-based processing. The execution will proceed from the initial state to the success state when the event Sig.Charging_Completed is processed. 


\subsubsection{Multi-threaded}

GiFT allows test engineers to create tests that are multi-threaded in nature. This gives the test engineer ultimate flexibility with test designs. To accomplish the visual multi-threaded specification, each test case may contain multiple flow charts. Each flow chart contains exactly one initial state to specify the beginning of that flow. At the start of a test, each flow is started by evaluating the transition out of the initial state and taking the transition, if appropriate. The system then context switches to the next flow and begins its execution until all flows have been started.

The ability to specify multi-threaded test cases gives test engineers the unique ability to generate input to the device in one flow while monitoring the reaction of the device with the second flow.

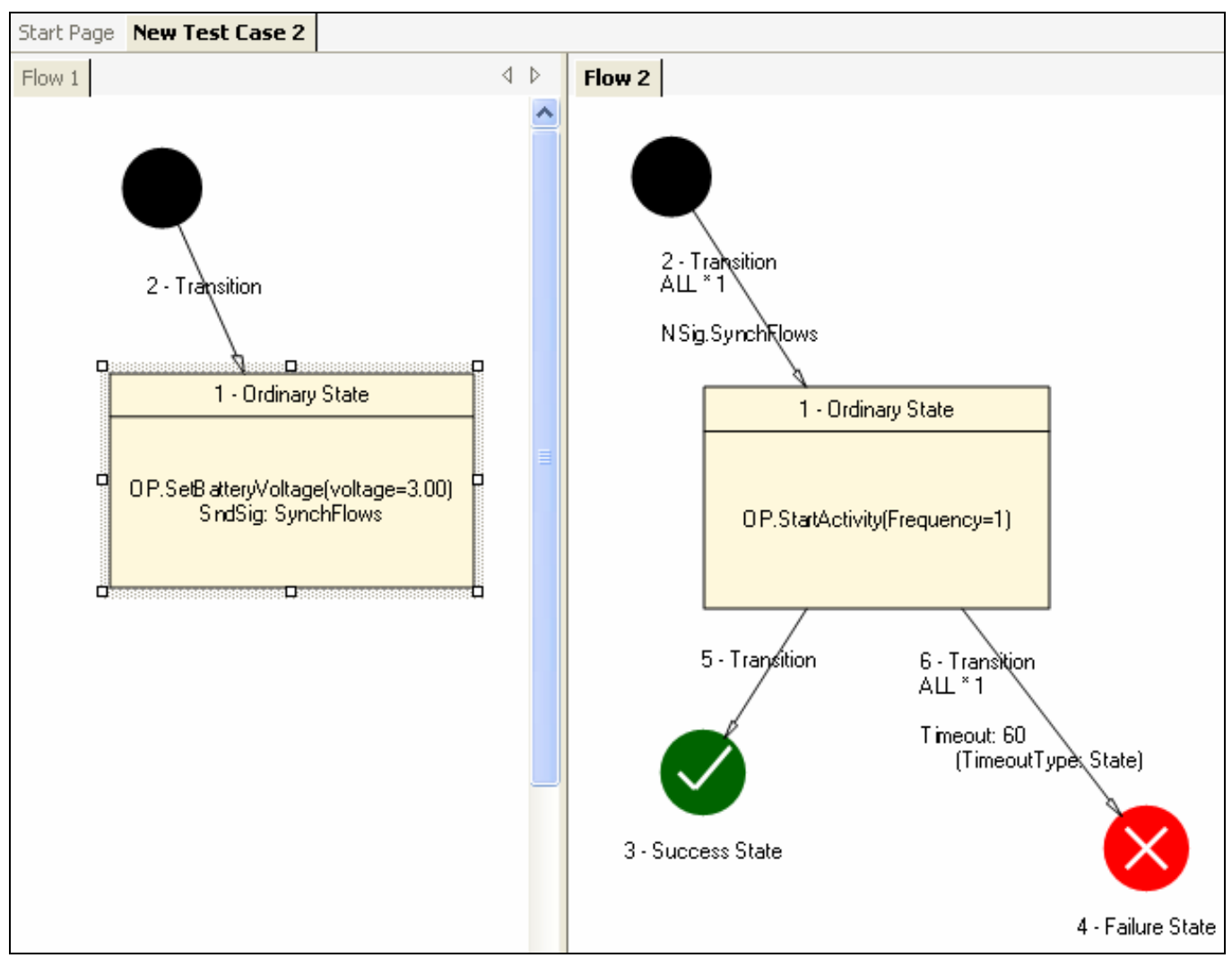

Figure 4.3: Multiple flows simulate a multi-threaded application 
There is also a means of communication among flows such that synchronization among flows may be attained. This is accomplished with a Named Signal. Named Signals are messages that are broadcast to each flow. To achieve synchronization, each flow can be in the initial state with a transition out that is waiting for the Named Signal to occur. To begin the test, the driving flow will issue the Named Signal. Upon receipt of the Named Signal by each flow, flow execution will begin. The same ability may be used at any point during the flow; its use is not limited to the beginning.

\subsubsection{Graphical}

One of the key elements of GiFT is that it is graphical in nature. The test engineer creates tests by using a graphical editor. The editor allows the specification of varying types of states and transitions. It also allows the editing of actions that occur within each state and the editing of conditions that may be present for a given transition out of a state. There is also a graphical editor for inputting expressions in a proprietary expression language.

In addition to the test design application being graphical in nature, there are other supplemental tools that are also graphical. After a test has run on the test station, the resulting log files may be used to graphically illustrate the path that was taken through the test flow. Another graphical tool uses an aggregation of multiple test run logs to show test coverage over a period of time. This activity ensures that each branch within the test has been taken at least once.

The graphical nature of GiFT is a great benefit to people involved in the testing process that do not have a background in software [20]. Each time a test is 
designed and implemented for a safety-critical application, the domain's governing body usually mandates a review of the test $[42,43]$. In each review, there should be representation from the Verification group, the Software group, the Systems group, and other organizations that are not solely based in software. For these groups, a test review is much more meaningful when they may inspect a visual representation of the test design instead of trying to decipher source code that is somewhat foreign to them. The foundation of GiFT's test designs is a flowchart, and because flowcharts are conceptually simple [20, 37], test designs in GiFT are easily comprehended.

\subsubsection{Randomized}

A core feature of the GiFT system is the ability to specify tests that execute in a pseudo-random nature. This feature is used mainly by the integration testing activity, but it is also useful for other testing types.

Randomization in GiFT works by allowing the test engineer to specify many various actions, decisions, and settings with some randomness to them. Randomization may be used in actions for setting values for variables. The random function allows setting a value that is within the valid range, a value equal to the max of the range, or a value equal to the min of the range. Decisions may also use randomness to guide a test down different pathways each time it is run. The action state may set a variable to a random value. The transitions out of that state may compare to the variable's value and the transition will be taken if the condition is met. For each execution, the actual path taken may be different. Initial test case settings may also utilize the randomization feature to set the 
embedded device into a random state before beginning a test. This is useful for verifying that an algorithm can identify behavior regardless of the initial state of the device.

\subsubsection{The Design is the Implementation}

One of the key features of GiFT is that the test design is the implementation. Traditionally, the test scenario is studied before designing a test plan. From the test plan, an actual test implementation is created. With GiFT, the test plan and test implementation are one and the same.

To achieve the goal of combining test design with test implementation, GiFT uses a proprietary file format. The output of the graphical test designer is a format that can be input and understood by the test engine. It is executable without any further intervention from the test engineer. This format is similar to a serialized object class that is then de-serialized by the test engine. The core data layer is shared between the test designer and test engine so that this serialization technique may be used.

By making the design and implementation one and the same, there is a great reduction in effort expended by the test engineer. There is also a reduction in flexibility. While this may seem like a negative effect, it is actually quite positive. With the increased rigidity, test engineers must conform to using the tools available to them and this keeps their designs within the bounds of a properly formatted test. Unlike $\mathrm{C}++$, they no longer have the freedom to perform actions outside of what is designated for them. This increases uniformity across tests. The increased rigidity also means that all of the low-level interface with the test 
station and the device are implemented only once in the test engine. Any deficiency discovered by a test engineer may be resolved once in the test engine with widespread benefit for all tests that are executed by the test engine in the future.

An additional benefit of having the design and the implementation be one and the same is that there is a guarantee that the design will always match the implementation. This is a rule that is not automatically enforced when using $\mathrm{C}++$ as a test implementation. With a $\mathrm{C}++$ implementation, minor adjustments to the test implementation may not always be reflected in the design since the design stage is generally finished when the implementation stage begins. 


\subsection{Test Designer}

The test designer is the application used by testing engineers to create visual tests. It is meant to be run directly on a user's desktop computer and is independent of the execution of the test. The following sections describe the test designer. Once the test design is complete, the test is moved to the test engine for execution.

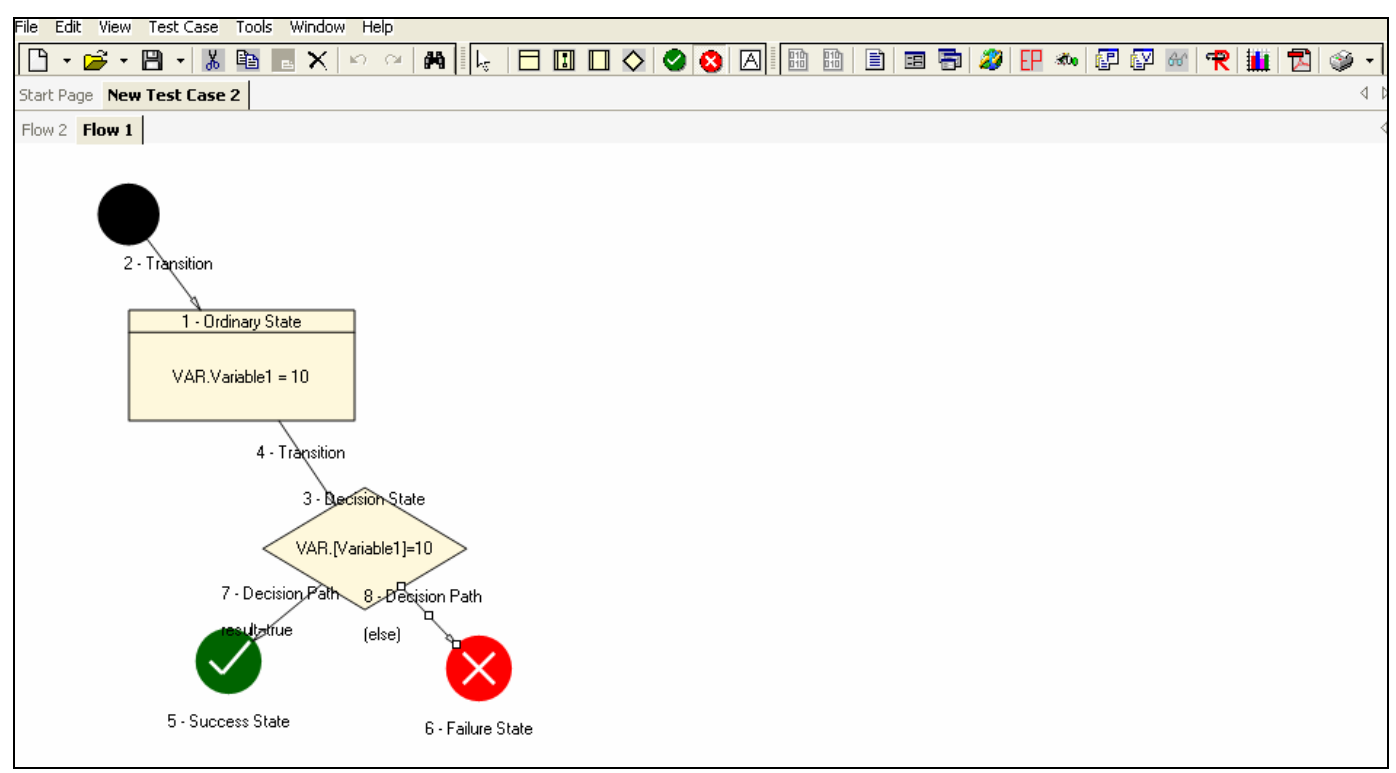

Figure 4.4: The Test Designer

\subsubsection{Document Types}

To implement the test system to be flexible enough for test engineers to fully express their test designs, GiFT has seven document types that it recognizes. The document types are Testcase, Macro, Testsuite, Configuration, Condition, Constant, and Formula. The document types all interact with each other to create a full test. 


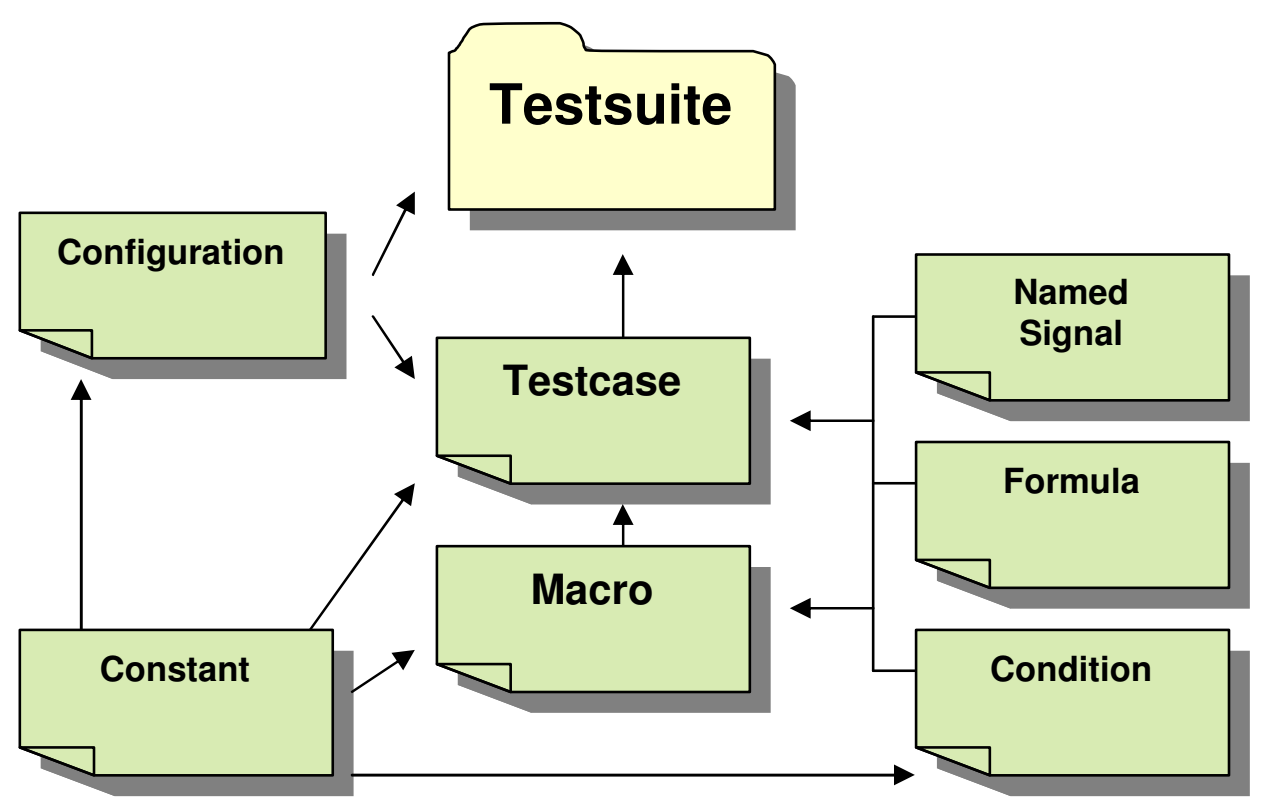

Figure 4.5: The Document Hierarchy

\section{Testcase}

A Testcase is the main document. It is one of two executable document types.

The Testcase may be standalone or it may include references to various components that it can import. A Testcase may include references to all of the other document types except a Testsuite. The Testcase is comprised of many components itself. These components include states, transitions, configurations, global handlers, etc. The Testcase must include at least one flow, one initial state per flow, and one terminal state per flow with a path between the initial state and terminal state. Optionally, the Testcase may include multiple flows to implement a multi-threaded Testcase. 


\section{Macro}

A Macro is very similar to a Testcase. The main difference is that a Macro is never run from within its own context; a Macro must always be invoked from within a Testcase or another Macro. As such, a Macro requires the use of a final state to indicate return of control to the parent when it is reached. A Macro also lacks the ability to set a configuration before execution since it is always invoked from an already executing state. The third large difference is that a Macro may only contain one single test flow. By specifying a piece of a Testcase as a Macro, it is easy to gain re-use out of a single common implementation; the Macro may be referenced by many different Testcases or other Macros.

\section{Testsuite}

A Testsuite is a collection of Testcases. The Testsuite is the other executable document type aside from a single Testcase. The Testsuite has properties of its own, including the number of times each Testcase should execute, the seed to feed into the randomization function, the configuration to set the system under test into before execution, and a set of actions that may be performed before, in between, and at the end of the set of Testcase executions.

\section{Configuration}

The Configuration document is similar to a settings file. The file is a large collection of parameters that may be programmed to the embedded system at any 
time before, during, or after a test. After designing the specific Configuration, it may be used within a Testcase or Testsuite.

\section{Condition}

The Condition document is a way to express a condition for a transition or a global handler. By creating a document to hold the Condition information, the same condition may be used in multiple places across many different documents without repeating work. The Condition document is used within Testcases and Macros.

\section{Named Signal}

The Named Signal is an object that is used for communication among flows. The Named Signal does not have any properties associated with it, but its role is very important. When there is a need for synchronization or message passing between flows, the Named Signal is the means to accomplish the task. The Named Signal is a document type so that more than one Named Signal may be created and used among flows. The Named Signal may be referenced by both Testcases and Macros.

\section{Constant}

The Constant document is similar to the Condition document because of its purpose: to reduce the amount of re-work within test designs. By defining the Constant once in a Constant document, the same Constant may be referenced in 
many test designs. If there is ever a need to change that Constant, the change need be made in only one central location. The Constant document may be used within a Testcase, Macro, Condition, or Configuration.

\section{Formula}

The last document type is a Formula. A Formula is a way to capture an expression that takes inputs to provide an output. Instead of having many different implementations of the same Formula in many different locations, the Formula is defined once and referenced in many places. If a change to the Formula is necessary, it is a single, central change instead of updating all the items that reference it. A Formula may be used within any expression.

\subsubsection{State Types}

There are many different states that may be used within a test flow. Each state type has its own properties and behavior. The state types are: Ordinary, Decision, Success, Failure, Final, Macro Invocation, and Testcase Reference.

\section{Ordinary}

The Ordinary state is the base state in a flowchart. It may be used to fork decisions, or it may be a state along a single path. The Ordinary state has the ability to execute a set of actions when it is reached. Upon test execution reaching an ordinary state, the actions are executed immediately without context-switching to other flows or listening for other events. Actions that may be executed in an 
ordinary state include setting values for variables, performing communication with the embedded device, and sending Named Signals to other flows.

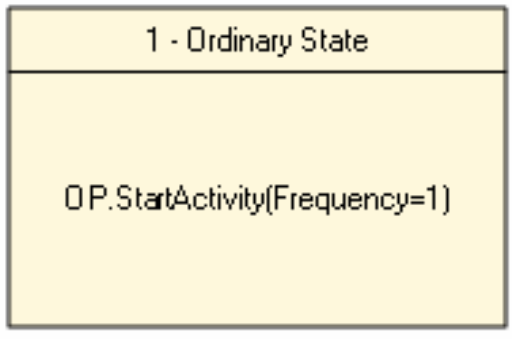

Figure 4.6: Ordinary state

\section{Decision}

The Decision state is central to the path of execution through the test flow. At the Decision state, comparisons are made. The comparison may be between variables or device values and any expression. The result of the comparison will direct the path of execution to one of the transitions out of the decision state. Which one it takes depends on the values for each transition and the result from the Decision state.

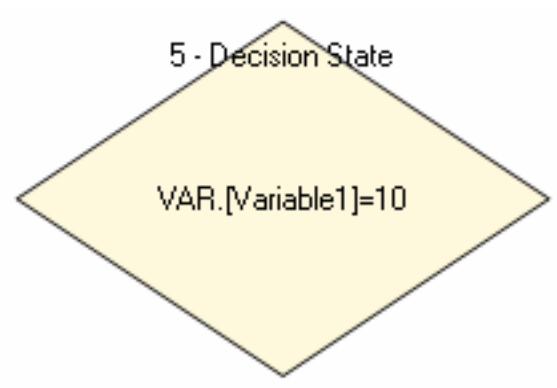

Figure 4.7: Decision state 


\section{Success}

The Success state is one of three terminal states. Upon reaching a success state in a Testcase flow, the test will end execution and report the test result as success. When reached within a Macro, the Success state will also end the test execution and report back a success result. When the Success state is reached within a Testcase Reference, the Success state will indicate that the referenced Testcase has completed successfully and the parent Testcase or Macro will continue execution without reporting any result of the test.

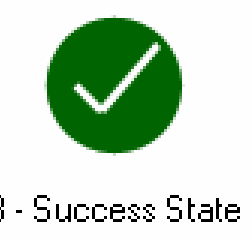

Figure 4.8: Success state

\section{Failure}

A Failure state may be used as either a terminal state, or a non-terminal state. In the case that there are no transitions out of the Failure state, the test will end and the overall status of the test will be failure, no matter which type of document was executing at the time. In the case that the Failure state has exiting transitions, the test result will be logged as failure, but the test will be permitted to continue. This is done to aid in information gathering and logging once a failure has been reached. After leaving a Failure state, there is no way to report anything but failure as the result, even if a Success state is later reached. 


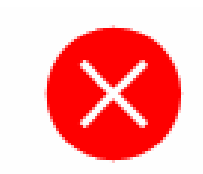

4 - Failure State

Figure 4.9: Failure state

\section{Final}

The Final state is a way to indicate to return execution from the executing flow to the flow which called it. This is only valid in a Macro and is used as a return statement since the Success and Failure states have different meanings.

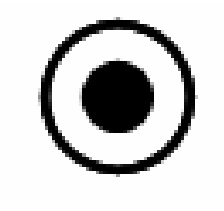

\section{1 - Final State}

Figure 4.10: Final State

\section{Macro Invocation}

The Macro Invocation state denotes the point at which to begin executing a referenced Macro. At the point that the Macro Invocation state is reached, all execution of the flow will stop and all signals will be ignored by the flow. Execution will begin in the referenced Macro and will not continue in the parent flow until a Final state is reached in the Macro. The Macro Invocation state allows for parameters to be passed to the Macro upon startup. This allows for communication between the parent and the Macro. 


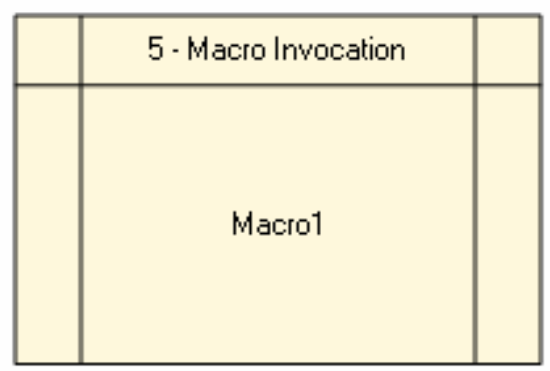

Figure 4.11: Macro invocation state

\section{Testcase Reference}

A Testcase Reference state is very similar to a Macro Invocation state except that it passes execution to another Testcase rather than a Macro. The difference is in the way that execution is passed back to the parent. In a Testcase, there is no Final state; instead, the Success state is used to indicate a return to parent when reached within the referenced Testcase.

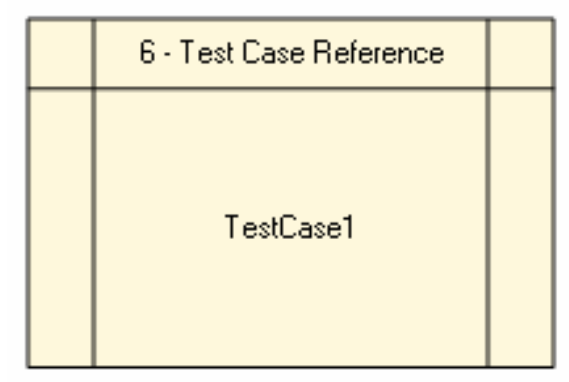

Figure 4.12: Testcase reference state

\subsubsection{Transition Types}

The links between states are called transitions. There are three types of transitions within GiFT: Completion, Condition, and Decision transitions. 


\section{Completion}

A Completion Transition is the simplest type of Transition. It is a single, unidirectional connection from one state to the next. It does not contain any information and is not waiting for any specific condition. As soon as the beginning state is reached, the Completion Transition will be taken without delay and the next state will be processed.

\section{Condition}

A Condition Transition is the most common type of transition used in test designs. The Condition Transition is also a single, uni-directional connection from one state to the next, but it contains a condition that must be met. Upon reaching the beginning state, the system is setup to listen for any information related to the Condition Transition. Only when the condition has been met, the transition will take place and transfer execution from the beginning state to the next state. After taking the Condition Transition, the system will stop listening for information related to the condition. The conditions placed on Condition Transitions may be comprised of a wait for certain values to be taken on by variables, a wait for a Named Signal to be sent, or a wait for a response from the embedded device.

\section{Decision}

The Decision Transition is only valid out of a Decision State. Likewise, a Decision State may only have Decision Transitions exiting the state. A Decision Transition is similar to a Condition Transition because it is only taken when the 
condition is met. However, a Decision Transition is usually a comparison that references the value in the Decision State. For example, the Decision State may contain the expression "True". The three Decision Conditions coming out of the Decision State would be "Result==True", "Result==False", and "Else". In this case, the Decision Transition with the expression "Result==True" would be taken and execution would flow to the state at the end of that transition. Each Decision State must have exactly one "Else" Decision Condition exiting from it. The "Else" transition is taken in the case that none of the other conditions are satisfied.

\subsubsection{Global Handlers}

Global handlers add flexibility for a test author. The Global Handler is an implementation of a watchdog on the test. Global Handlers are specified similarly to conditions and they are associated with a set of actions to take if the specified condition is met. The Global Handler is always active and will execute at any time the condition is met within the scope of the testcase or macro of which it is a part. Global Handlers add the important ability to monitor for error conditions or situations that must be triggered upon.

\subsubsection{Expression Parsing}

To implement comparisons and assignments, there is a need to specify expressions within GiFT. It is desirable to give the test authors the flexibility that they had when designing and implementing tests in $\mathrm{C}++$. To this end, a custom expression parser was developed.

The expression syntax is very similar to $\mathrm{C}++$. The operators all follow very closely to keep confusion and relearning to a minimum. Once the expression is 
specified, it is necessary to interpret the expression and execute the actions specified. Because the test engine is operating in real-time and the test designer is not, it is desirable to have as much processing on the test designer side as possible. To accomplish this, the expression parsing on the test designer not only checks for syntax, but it also builds a parse tree and stores it in the Testcase file. When the test engine comes across an expression during execution, it merely operates on the already defined parse tree. This extra step removed from the test engine aids in meeting performance requirements.

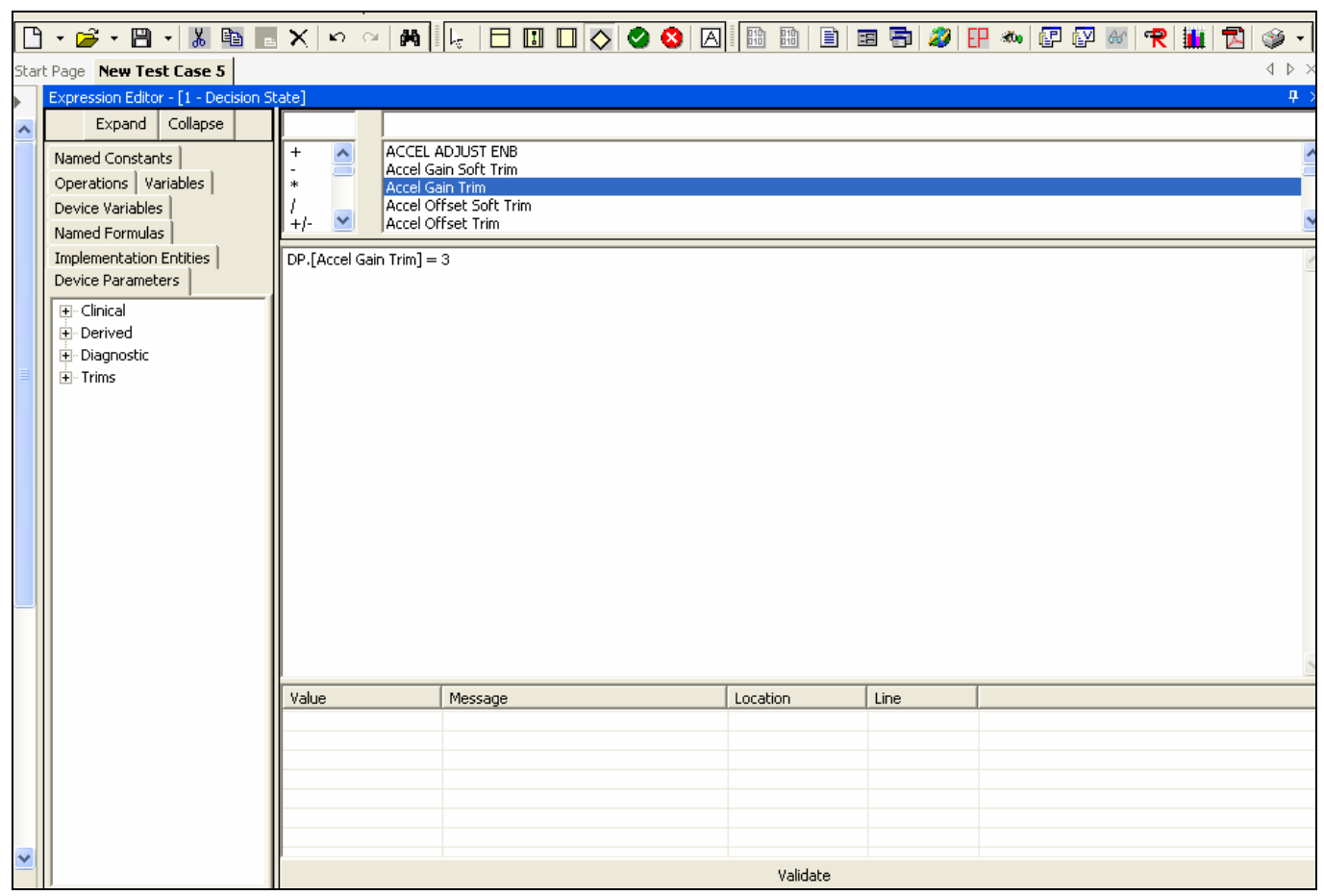

Figure 4.13: Custom expression editor

\subsubsection{Storage Format}

To support the communication between the test designer and test engine, there is a need to express every detail of a test from the test designer in such a way that it may be easily reinterpreted by the test engine. To accomplish this, the storage 
format used is XML. The XML format is used for all document types. By using $\mathrm{XML}$, it is straightforward to express the test documents in a way that may be readily created by the test designer and readily consumed by the test engine. The layout of the XML files closely resembles the object structure defined in the Data Layer of the system. In this regard, the saving and reading of document types is similar to serializing the object structure on the test designer and de-serializing it on the test engine. The XML transport paradigm has worked well for communication between the two applications and it has also lent itself well to the storage and maintenance of test objects by the testing teams.

\subsection{Test Engine}

The test engine is where the execution of tests takes place. The test engine is the topmost piece that sits on a layer of many supporting tools. It is a controller that moderates the behavior of the test specification while making calls to outside tools and deciding which actions to take based on the responses from those tools.

The test engine works by first loading up the test files and external files necessary to interface with the system. It then sets the environment into a known state, runs some sanity checks on the system, and begins test execution. The test execution phase involves first setting the state of the system as specified then initializing each flow of the testcase. Once each flow is initialized, the test engine is responsible for executing any actions in the testcase by calling out to external tools and capturing the return information from the tools to operate on. The test engine receives the input from the tools, makes evaluations against the conditions 
in the Testcase and moves to the next state of execution in the testcase flow, if appropriate. The test engine will finish executing when the state of execution reaches a terminal state, the testcase timer expires, or there is an error encountered.

\subsubsection{Preprocessing}

Before any test execution may occur, the Testcase is run through a preprocessing method. The need for pre-processing is twofold: it checks the Testcase for errors and it limits the processor-intensive activity to a period in time when the process does not need to perform in real-time alongside the device under test.

The pre-processing method first checks the Testcase to make sure that the test contains no validation errors. This check helps to reduce errors during execution that may cause run-time problems and even put the test system into an irrecoverable state. The pre-processing check ensures that all necessary references are available, that there are no datatype mismatches, and that the flows meet a set of minimum requirements such as having exactly one initial state and a path between it and a terminal state. After the validation check, the pre-processing method takes care of loading all of the necessary references so that there is no disk I/O during test execution. All of the necessary supporting objects are created in memory and then the test execution may begin.

\subsubsection{Flow Processing and Event Processing}

After the pre-processing is finished, the test engine sets up the environment and begins the test execution phase. During this phase, there are two main activities that are being performed: flow processing and event processing. 
Flow processing begins by preparing the flows for execution. Each flow's initial state is set as the current state. The state to which it is attached is set as the next state. If there is a condition on the transition, the pieces of the condition are registered with the test station so that the event will be seen when it happens. Once the initial states of all the flows are ready, the test engine begins the flow processing loop.

There is a limitation that the test execution may not utilize Windows' multithreaded ability for each flow; this is due to several factors involving the architecture of the test station and its critical timing information. Because of this, the test engine must simulate multi-threaded behavior using one Windows thread. This is accomplished with a round-robin approach. The flow processing loop goes one-by-one to each flow and processes it. During the processing, the flow is checked to see if there are any events that have been delivered to it. If there are, the events are checked against the currently waiting transition. If the condition is met, the transition is taken. The "next state" becomes the "current state" and the connected state moves into the "next state" spot. If there is a completion transition out of the new "current state" then the transition is taken immediately and the states and conditions are updated again. Any new conditions are registered and the actions in the new "current state" are executed. Once the conditions are registered and the actions have been performed, the test engine moves on to evaluate the next flow. This continues until a terminal state in any flow becomes the "current state." 
Despite the restriction on using Windows' multi-threaded ability for each flow, there is no such restriction in using new threads for utility functions within the test engine. This allows a separate thread to run for the event processing loop. The event processing loop is a loop that monitors a queue of incoming events and delivers them to the appropriate place. When each flow registers for a condition, the event processing loop is notified that the particular flow is interested in the event. When the event arrives, the event processing loop will copy the event to each flow that has registered for it. That is the sole function of the event processing loop. When the flow processing loop eventually processes the flow, it will recognize that new events have arrived and will evaluate each pending condition against the new events.

While there is some room for improvement in the Flow and Event processing activities, they are currently working quite well within the constraints of the system. The events are processed and delivered in a timely manner and the flow state is able to keep up with the speed of the device under test.

\subsubsection{Tools}

The following sections will describe the tools that the test engine relies upon:

\section{Test System}

The test engine operates on a test system platform. The platform hosts two PCs, a breadboard mockup of the embedded device, a logic analyzer, a digital interface to the breadboard, a communication interface to the breadboard, and a tool to provide stimulus. The PCs on the cart control all of the various tools and 
provide a unified application programming interface to communicate with them all. The test engine executes on one of the PCs on the test platform and directly uses the application programming interface (API) to make calls to all of the tools available.

\section{Digital Interface}

The digital interface is a primary component of the test platform. The digital interface is like a window into the behavior on the breadboard. It is extremely useful for white box testing since the only alternative is a logic analyzer. The digital interface can be set up in a way to watch any specific region of the firmware and to only trigger on specific conditions. These conditions can be simple "ands" and "ors" or they can be more complex combinations of conditions. By using the digital interface, the test engine can watch for the conditions to occur that relate to the condition on a transition within the test flow. Upon satisfaction of the condition, the test engine is notified and takes the appropriate behavior. Without the digital interface, the ability to wait for conditions would be severely limited. The digital interface gives great flexibility and power to the authors of GiFT tests.

\section{Communication Interface}

The communication interface is another primary component of the test system. The digital interface and communication interface are both responsible for communicating with the breadboard mockup of the embedded device. The 
difference between the two is that the digital interface is merely a window into breadboard activity and may not be used to send information to the device while the standard communication interface is very similar to the final communication interface of the shipped embedded system and may be used for bi-directional communication.

The test system employs the standard communication interface to accomplish nearly all communication with the device. GiFT allows users to specify the sending and receiving of communication with the device. To accomplish the implementation of this communication, the test engine uses the communication interface to execute the commands that are available to the test authors.

\section{Stimulation Tool}

The stimulation tool is another important component of the test system. When using breadboarding instead of simulating for a testing methodology, there is a need to create inputs for the breadboard to respond to. The purpose of the stimulation tool is to simulate input to the embedded device.

In testing, one of the most important activities is providing input to a system and observing the output. The stimulation tool allows test authors to provide such input by implementing an application programming interface. The test author may specify various inputs during design time. When the test engine encounters the need to simulate activity, it will make a call to the stimulation tool through the API. The stimulation tool will, in turn, drive inputs into the breadboard while the 
test engine is observing the behavior through various other means such as the digital interface and communication interface.

\subsection{Test Simulator}

While the test engine is well-suited for execution, it is sometimes difficult to use during development of a test. This is due to the lengthy time it takes to initialize the test system, the overhead of running the test on the test platform, and the inability to examine the test until it is completely finished.

To aid with the development of GiFT test design, the test engine simulator was created. The test engine simulator is a piece of software that may be run alongside the test designer. It completely emulates the test engine without the overhead of running the test on an actual test system. In addition, the output of the test engine simulator is given immediately after each step so that the test developer may better understand the inner workings of the test. Instead of being driven by a device or other system tools, the test engine simulator is driven by the user. After loading the Testcase, the simulator will start the test flows. At the points where it reaches a waiting condition, the test engine simulator allows the user to simulate input to the system. When the user provides the simulated input, the test will continue until the next condition, just as the test would do when running on the real test engine.

The benefit of providing a test engine simulator becomes apparent when test authors need to debug through the logic in their tests. After the logic is verified, 
the test author has eliminated one possible risk factor before running the test against the real device.

\subsection{Post-processing}

In the case that a Testcase passes, there is often no further need for analysis. However, when a test fails, there must be a good support system for investigating the failure. To this end, the GiFT tool has implemented a key component called Playback.

Playback is a post-execution visual representation of the test execution. Because the test system must maintain a high level of processing power to keep up with the real-time embedded device, there is not enough computing power available to show diagnostic or debugging information during the test execution. During execution, logs are created in memory. To prevent slow disk I/O during test execution, the log files are written to disk after the execution completes. The problem for test engineers is that the log files are often cryptic and difficult to understand. In addition, the visual aspect of GiFT is lost if the test engineers are required to wade through pages of cryptic logs to diagnose a failed Testcase. Playback is a tool that addresses these issues.

Playback consumes the log files that were created during a test execution as well as the original Testcase file. Once the files are loaded, test authors are given a visual representation of their Testcase, similar to the view available in the test designer. The Playback tool operates similarly to many debugging applications. Users may step through the execution of a test one step at a time to analyze the 
path that was taken during execution. The Playback tool also provides some information about the information received from the device during the test to explain the reasoning behind taking a certain path.

Playback is a powerful tool for the test authors to visually interpret the behavior of their tests during execution. It is not only a great diagnostic tool, but also a great tool to use during the test development phase. 


\section{Chapter 5}

\section{Evaluation}

Integration testing has been the main driver of the requirements for GiFT. Because integration testing requirements for a testing tool are usually a superset of the requirements for other testing approaches [7], GiFT is easily used for both integration testing and other less-intensive testing activities. This has caused the outcome to be extremely well-suited for integration testing without sacrificing the usability for other testing approaches.

GiFT has been fully implemented and deployed for evaluation. It has been deployed for unit testing, verification testing, integration testing, ad-hoc testing, and regression testing. While these different activities have all utilized GiFT at least minimally, the largest focus has been on integration testing and verification testing. This evaluation will focus specifically on these two types of testing activities. 


\subsection{Strengths}

The following list illustrates the flaws that exist in the typical approach to integration and verification testing. Each of these flaws is addressed with the introduction of GiFT.

\section{Test Engineers must have Low-level Knowledge of the Test System}

GiFT has completely removed the low-level detail from the user by directly executing the user's graphical test design. The user is only concerned with the graphical layout of the test design and it is automatically translated into implementation by the test engine. All of the low-level details are implemented one time only in the test engine and are hidden from the end users.

\section{Test Maintenance}

An important evaluation criterion when comparing testing approaches is the amount of maintenance required to the repository of developed tests when the device changes. Ideally, a change in the device is transparent to the test engineer and the test will continue to execute properly. With the typical testing approach, the test calls out specific implementation details that may change and require an update to the test. For example, if the maximum value of a variable called abc is 100 , the test would manually call an instruction to set abc $=100$. With the advent of GiFT, the details of the variables are all dynamically loaded. This means that there is a call to set $a b c=\max$. If the maximum value changes in the next release 
of firmware to 101, the update is handled automatically and no intervention is required by the test authors.

\section{Limited Re-use}

The typical code written for integration and verification tests is written in such a way that re-use is very limited. There are no pieces that can easily be shared across multiple testcases; each testcase has to re-implement every part of a testcase from top to bottom. While the Guidant approach to verification testing solves this problem minimally by introducing re-usable templates, it doesn't gain all the benefits of having re-usable components. GiFT solves this problem by introducing macros, embedded testcase references, named configurations, and named conditions. By allowing such flexibility, some testcase designs are as simple as making calls to pre-defined building blocks and evaluating the result. In the other approaches, there is an enormous amount of copying and pasting. This becomes an even larger gain for GiFT because modifying a simple macro used in many places affects each of the testcases that uses it with only one edit in one location. With the other approaches, a widespread change could mean modifying thousands of testcases individually.

\section{Manual Intervention to Capture Results}

The typical approach to both integration and verification testing relies heavily on the logic analyzer to capture results of the testcase. This requires manual setup of the logic analyzer to trace the variables of interest. When the firmware 
changes, the locations change and the logic analyzer setup must be changed accordingly. In GiFT, the details are removed from the user. By using the digital interface, the need for the logic analyzer is eliminated. The digital interface is context-aware such that names within the GiFT test will automatically link to the proper firmware locations, even after a rebuild of the firmware. This eliminates the need to manually modify logic analyzer setup files to run tests.

\section{Manual Interpretation of Results}

Once the typical integration and verification tests are run, the output of the tests are logic analyzer trace files. These files are manually interpreted to determine if the conditions match the expected results to indicate a passed testcase. A more advanced common approach is to use a tool to automatically process the results. This is an improvement, but the necessary post-processing is not eliminated. In GiFT, there is no need for post-processing. The test runs in real-time and the result is also determined in real-time. When the test branches to a success state, the test result is success; when the test branches to a failure state, the test result is failure. The result is immediately known upon completion of the test run.

\section{Difficulty Debugging}

Embedded systems tests are notorious for being difficult to debug. Because the test runs in real-time, the only debugging tool available is usually a re-run of the test with additional information captured by the logic analyzer. The test 
engineer then reads cryptic logs and tries to interpret them. The debugging ability in GiFT is a stark contrast to this manual troubleshooting activity thanks to extensive logging and an after-the-fact playback tool. The tool allows the user to step through the results of the test execution with a visual representation of each action taken, each decision evaluation, and each branch taken in the test flow. This is a powerful tool to debug both the test and the firmware by comparing the expected results with the actual results from the test run.

\section{Learning Curve}

A new test engineer has a lot to learn when joining an embedded systems testing group. The test engineer must learn device knowledge, embedded testing methodology, domain knowledge, and test system knowledge. By using GiFT, the need to learn test system knowledge is eliminated and the newly hired test engineer can focus on the more critical aspects of effective testing. In fact, due to the elimination of implementation, newly hired test engineers need not have any knowledge of $\mathrm{C}++$. Because of this aspect of GiFT, it would be easy for a testing group to open the door to many other disciplines outside of software engineers. To acquire employees with the application knowledge required for effective testing, the testing group now has the ability to hire domain experts and testing experts rather than software experts. GiFT also shifts the focus of the testing activity from software development to effective testing practices. 


\section{C++ is not Readable and Understandable by Everyone}

A large part of the testing process is the review of test designs by groups outside of the testing organization. These groups may include systems engineers, domain engineers, or even regulatory oversight. The involvement of outside groups adds value to test design reviews due to their particular areas of expertise.

While the attendance of outside groups is beneficial in a test review, they are only helpful when they have an understanding of the contents of the review. In the case of the typical approach, the usefulness of outside groups reviewing implementation is limited because members of the outside groups are not software engineers and generally do not understand $\mathrm{C}++$ code. While they are effective in reviewing the design of the test, the implementation is not covered. With GiFT, the design and implementation are one and the same. This makes a test review very easy to understand and eliminates the need for a test implementation review. The elimination of a test implementation review is a large time savings for everyone involved in the review process. In addition, the GiFT test designs are graphical in nature and will often lead to higher understanding of the test design than a procedural text description of a test design.

\subsection{Weaknesses}

While GiFT has many advantages over the other testing approaches, there are also a handful of disadvantages. Some of these disadvantages are common to all available testing approaches, while others are unique to GiFT. They are illustrated below: 


\section{Digital Interface Performance}

To ease information passing between the test station and the system under test, GiFT relies heavily on the digital interface to the device. Due to speed and hardware limitations, the particular implementation of the digital interface used for the evaluation of GiFT was only able to monitor up to 120 different firmware locations. In verification tests, this is often adequate because of the generally smaller size of verification tests. In integration tests this limitation has been a problem, but only in a few, limited cases. The alternative testing approaches also utilize the digital interface and therefore have a similar limitation. All approaches using the digital interface have a significant advantage over the previous approaches that used the logic analyzer because the logic analyzer only provided the ability to monitor 16 locations at a time.

\section{System Speed}

To test a non-emulated real-time device, the test system must be able to keep up with the real-time device and not fall behind in its execution. This becomes a limitation when there is a high traffic load placed on the communication interface between the device and the test system. When the digital interface has a large number of locations to monitor and the locations are commonly updated, there is an enormous amount of communication that must happen between the device and test system. In some cases, the test system is unable to process all of the events before the next execution step of the device. In this case, the test system and device are out of sync and the test will not execute appropriately. Because of this 
limitation, there is an artificial limit on the digital interface in GiFT to keep the amount of communication low enough to be processed in real-time.

\section{Culture}

One of the most significant challenges that GiFT faced in the evaluation is not technical in nature, but rather cultural. In the testing organization used to evaluate the paradigm, $\mathrm{C}++$ has historically been used as the tool to implement tests. Due to this, the hiring paradigm for the testing organization has been to recruit software engineers that know how to use $\mathrm{C}++$. Over time, this has created a large testing organization comprised mainly of software engineers who enjoy software design and implementation. With the typical approaches to testing, the software engineers are able to exercise their software design and implementation skills. Because GiFT is mainly a design tool where the design is also the implementation, it removes the test authors from the activity of software design and implementation. Being that the primary evaluators of GiFT were software engineers that enjoy software design and implementation, they were displeased by using a tool that removes them from the ability to exercise the skills which they enjoy.

\section{Process}

Another significant challenge that stems from the culture of the evaluating testing team is related to their process. Historically, because design and implementation of $\mathrm{C}++$ tests are two different entities, the team is used to having 
a textual description of a test design before implementing the design. Although GiFT integrates the design and implementation into one graphical object, the testing team still desires a textual test design before moving on to the graphical test design. The philosophy of the GiFT design is to reduce workflow by combining the two activities of design and implementation into one, but the testing team did not realize this gain because of their insistence to have a textual design before a graphical design.

\section{Design is the Implementation}

While there are significant advantages to having the design and implementation be one and the same, there is also a subtle weakness to the approach. When tests are initially designed, they must go through a review process. Because there is no need for implementation with GiFT, this is the only review needed. However, when the test engineer executes the test design on the test station, it may be realized that there are changes that must be made to the test design. With $\mathrm{C}++$ implementation approaches, this is not a problem because it is the implementation that changes, not the design. With GiFT, to affect the implementation, the test engineer must modify the design. This subtle difference means that the design review is now invalid and must be re-reviewed. This may be solved with a process change to have the GiFT test designs reviewed after the test is successfully executed on a test station, but it then prohibits the gains achieved by having reviews early in the design process. In the end, this may not be too costly since the gains achieved by having early reviews usually end up 
being gains in the implementation phase; with GiFT, there is no implementation phase.

\subsection{Speed of Implementation}

To answer whether GiFT or the common testing approach is faster for a test engineer to use, one must consider multiple factors: history, technical aptitude, and the test engineer's experience with each approach.

History plays a big part in a test engineer's speed using both GiFT and the typical approach. The evaluating test engineers were all familiar with the typical testing approach and unfamiliar with GiFT. As such, they are slower using GiFT because it requires learning a new paradigm.

Technical aptitude has a lot to do with speed. For a test engineer who doesn't have a strong background in $\mathrm{C}++$ or a strong familiarity with the test library and test station, a majority of their time using the typical approach is spent debugging intricacies with the library and the test station. For this type of user, GiFT is faster because those implementation details are removed from the equation. For a test developer who is very familiar with the technical intricacies of the library and test station, the $\mathrm{C}++$ approach is often faster.

The previous experience of the test engineer plays a large part in determining whether GiFT or the $\mathrm{C}++$ approach is faster. For a test engineer very familiar with the $\mathrm{C}++$ library, it is the faster approach; for a test engineer familiar with GiFT, it is the faster approach. It has been found that for a test engineer with no 
background in either tool, GiFT is a faster approach, despite the test engineer's general understanding of $\mathrm{C}++$.

Because of the difficulty for an accurate comparison between the two approaches, it is difficult to conclude with any certainty that either approach is faster than the other in terms of initial implementation time. However, speed of implementation is only one of many factors that contribute to the overall effectiveness of each approach.

\begin{tabular}{|l|c|c|}
\cline { 2 - 3 } \multicolumn{1}{c|}{} & GiFT & C++ \\
\hline $\begin{array}{l}\text { Speed of } \\
\text { Implementation }\end{array}$ & Undetermined & Undetermined \\
\hline Learning Curve & $\begin{array}{c}\text { Quick - Visual and } \\
\text { Flowchart Based }\end{array}$ & $\begin{array}{c}\text { Slow - Must Learn } \\
\text { Custom C++ Library }\end{array}$ \\
\hline Ease of & Playback Tool & $\begin{array}{c}\text { Must Decipher } \\
\text { Cryptic Logs }\end{array}$ \\
\hline Pebugging & Provides Visual & Display \\
\hline Review Process & Updated & Requires Manual \\
& Automatically & Updates to Many \\
& Flowcharts are & C++ is not Universal \\
\hline Culture & Universally & \\
\hline & Software Engineers & Software Engineers \\
& Dislike Using & Prefer Writing Code \\
& Graphical Approach & \\
\hline
\end{tabular}

\begin{tabular}{|c|}
\hline Advantage \\
\hline Undetermined \\
\hline GiFT \\
\hline GiFT \\
\hline GiFT \\
\hline GiFT \\
\hline C++ \\
\hline
\end{tabular}

Figure 5.1: GiFT vs. Typical Approach Comparison 


\section{Chapter 6}

\section{Future Work}

While GiFT has proven to be an effective testing approach for many various types of testing activities, there are still opportunities for improvement. The potential improvements include moving to a real-time operating system, moving to a more powerful and re-designed test system, modifying it for even broader testing use, implementing numerous GUI enhancements.

Currently, the test engine executes on a test system that runs Windows as its operating system. Due to the fact that GiFT executes on the Windows operating system, there is a lack of the ability to have complete control over the test station. It has been noted several times that Windows will execute a processor-intensive task during a time-critical portion of a test execution. These cases cause nondeterministic behavior to be exhibited by the test system. In the future, it would be worthwhile to experiment with a real-time operating system to eliminate the nondeterministic behavior and gain complete control of the test station.

The test station used for evaluation of GiFT is a conglomeration of many individual components that were independently designed before being melded together. The lack of a central system design has led to some deficiencies that 
would be worthwhile to address during a test station re-design. At the same time, the system could be designed to benefit from the latest dual and quad core technology. With a proper test system design in place and the latest technology, GiFT could potentially increase the amount of information from the device that it is able to process. This will further empower test engineers with more test design flexibility.

GiFT is currently focused on testing embedded devices. While firmware testing has been the focus, there exist many additional products that may benefit from using GiFT as a testing approach. For example, many embedded devices have external instruments that communicate with the embedded device. The testing of these communication devices is a large effort that could potentially benefit from using GiFT if the right modifications are put into place to enable GiFT to interface with it.

During the development of GiFT, the paradigm was always to focus mostly on the functionality of the system. This development process has resulted in an application that works very well, but it also leaves room for improvement to the graphical user interface. Improving the usability will make the experience of using GiFT much more pleasant and potentially more effective.

While GiFT itself has some areas for improvement, there is also room for improvement in its evaluation. GiFT has been thoroughly compared against the common testing approach, but it has not been compared against other cutting-edge testing techniques. It would be worthwhile to rigorously evaluate GiFT against other innovative testing approaches. 


\section{Chapter 7}

\section{Concluding Remarks}

The Graphical interface Flowchart Tool (GiFT) approach has shown to be effective in improving testing, especially related to the areas of integration and verification testing. The common testing paradigm and the approach to verification testing at Guidant both have significant downfalls that are addressed and improved upon with GiFT.

GiFT is a unique approach to testing software because it isolates the test engineer from software implementation details. The evaluating testing group is mainly comprised of software engineers; the departure from test implementation is looked at as a detriment because of their fondness of software development. The reason for hiring software engineers into the testing group is historically due to the fact that the software engineering skill set was necessary to implement test designs. The GiFT tool has eliminated the need for test engineers to possess software engineering skills. With this new approach, testing organizations have the opportunity to become more effective because they are no longer limited to hiring software developers as test engineers. With GiFT, testing organizations have the ability to hire testing experts or domain experts to perform testing that 
may add an additional level of testing performance not possible with a software engineer.

The first version of GiFT has proven to be an effective testing tool. Whether it will overcome cultural barriers and gain broader acceptance still remains to be seen. 


\section{Bibliography}

[1] Altman, Lawrence K. "Heart Device Recall Poses a Quandary for Patients." New York Times 16 October 2007.

[2] Auriga, Inc. Testing of Embedded Software Products. Auriga, Inc. home page. 2 Nov. 2008 $<$ http://www.auriga.com/files/projects/Auriga_test_of_emb_sw_prod.pdf $>$.

[3] Bajaj, Vikas. "F.D.A. Puts Restrictions on Guidant." New York Times 28 December 2005.

[4] Balakrishnan, Subhashini, and Sofiene Tahar. "A Hierarchical Approach to the Formal Verification of Embedded Systems Using MDGs." In Proc. of the Ninth Great Lakes Symposium on VLSI (1999): 284.

[5] Balarin, Felice, et al. "Formal Verification of Embedded Systems based on CFSM Networks." DAC '96: Proceedings of the 33rd annual conference on Design automation (1996): 568-571.

[6] Beizer, Boris. Software Testing Techniques. $2^{\text {nd }}$ Ed. New York: Van Nostrand Reinhold, 1990.

[7] Beizer, Boris. Black-Box Testing. New York: John Wiley \& Sons, Inc., 1995.

[8] Beyeda, Sami, Volker Gruhn, and Michael Stachorski. "A Graphical Class Representation for Integrated Black- and White-Box Testing." In Proc. of the IEEE International Conference on Software Maintenance (2001): 706.

[9] Boniol, Frederic, Virginie Wiels, and Emmanuel Ledinot. "Experiences in using model checking to verify real time properties of a landing gear control system." Presented at the European Congress ERTS, 2006.

[10] Broekman, Bart, and Edwin Notenboom. Testing Embedded Software. London: Pearson Education Limited, 2003. 
[11] Broy, Manfred. "Requirements Engineering for Embedded Systems." 1997. Technische Universität München, Institut für Informatik. 19 Oct. 2008 $<$ http://www4.in.tum.de/publ/papers/femsys_boesswet_1997_Conference.p df $>$.

[12] Burnstein, Ilene. Practical Software Testing. New York: Springer-Verlag, 2003.

[13] Dasdan, A., D. Ramanathan, and R. Gupta. "An interactive validation methodology for embedded systems." In Proc. High-Level Design Validation and Test Workshop (1997): 123-130.

[14] Devaraj, George, Mats P. E. Heimdahl, and Donglin Liang. Condition based coverage criteria: To use or not to use, that is the question. 2005. U of Minnesota, Minneapolis. 19 Oct. $2008<\mathrm{http}$ ://wwwusers.cs.umn.edu/ devaraj/paper.pdf $>$.

[15] Easterbrook, Steve, and John Callahan. "Formal Methods for Verification and Validation of partial specifications: A Case Study." Journal of Systems and Software 40 (1998).

[16] Encontre, Vincent. "Testing embedded systems: Do you have the GuTs for it?" The Rational Edge (2003). 19 Oct. 2008

<http://www.ibm.com/developerworks/rational/library/459.html>.

[17] Fowler, Kim R. What Every Engineer Should Know About Developing Real-Time Embedded Products. Boca Raton, Florida: CRC Press, 2008.

[18] Goddard, Steve. "Analyzing the Real-Time Properties of a Dataflow Execution Paradigm using a Synthetic Aperture Radar Application." In Proc. of the 3rd IEEE Real-Time Technology and Applications Symposium (1997): 60.

[19] Gustafson, David A. Schaum's Outline of Theory and Problems of Software Engineering. New York: McGraw-Hill, 2002.

[20] Hammer, Eric M. Logic and Visual Information. Stanford, California: CSLI Publications, 1995.

[21] Harrison, Neil B. "Testing: It's Part of Your Job - Make it Effective!" Presented at the International Conference on Object-Oriented Programming, Systems, Languages and Applications (2007).

[22] Heitmeyer, C., et al. "Tools for Formal Specification, Verification, and Validation of Requirements." In Proc. 12th Annual Conf. on Computer Assurance (1997): 35-47. 
[23] Holzmann, Gerard J., and Margaret H. Smith. "Automating software feature verification.” Bell Labs Technical Journal (2005): 72-87.

[24] Horowitz, Paul, and Winfield Hill. The Art of Electronics. Cambridge [England]: Cambridge University Press, 1989.

[25] Jahanian, Farnam. "Run-Time Monitoring of Real-Time Systems." Advances in Real-time Systems. Ed. Sang H. Son. New Jersey: Prentice Hall, 1995. 435-460.

[26] Jorgensen, Paul. Software Testing: A Craftsman's Approach. $3^{\text {rd }}$ Ed. Boca Raton, Florida: Auerbach Publications, 2008.

[27] Larsen, Kim G., Marius Mikucionis, and Brian Nielsen. "Online Testing of Real-time Systems Using Uppaal.” Formal Approaches to Software Testing 3395 (2005): 79-94.

[28] Larsen, K. G., et al. "Testing real-time embedded software using UPPAALTRON: an industrial case study." In Proc. of the 5th ACM international Conference on Embedded Software (2005): 299-306.

[29] Loveland, Scott, et al. Software Testing Techniques. Hingham, Massachusetts: Charles River Media, 2005.

[30] Marwedel, P. Embedded System Design. Secaucus, New Jersey: SpringerVerlag New York, Inc., 1986.

[31] Meier, Barry. "Citing Flaws, Maker Recalls Heart Devices." New York Times 18 June 2005.

[32] Myers, Glenford J. The Art of Software Testing. $2^{\text {nd }}$ Ed. Hoboken, New Jersey: Wiley, 2004.

[33] Perry, William E. Effective Methods for Software Testing. $2^{\text {nd }}$ Ed. New York: Wiley, 2000.

[34] Perry, William E. How to Test Software Packages. New York: Wiley, 1986.

[35] Pike, L. Formal Verification of Time-Triggered Systems. Diss. Indiana U, 2006. UMI: AAI3215296.

[36] Sommerville, Ian. Software Engineering. International computer science series. Boston: Pearson/Addison-Wesley, 2004. 
[37] Staunstrup, Jorgen, et al. "Practical Verification of Embedded Software." Computer 33.5 (2000): 68-75.

[38] Steiner, Neil, and Peter Athanas, "Hardware-Software Interaction: Preliminary Observations." In Proc. of the 12th Reconfigurable Architectures Workshop, RAW (2005).

[39] Tan, Li. Evidence-Based Verification. Diss. State U of New York at Stony Brook, 2002. Proquest UMI, 2002. 764991991.

[40] Tan, Li, Oleg Sokolsky, and Insup Lee. "Specification-based Testing with Linear Temporal Logic." In Proc. of the 2004 IEEE International Conference on Information Reuse and Integration (2004): 493-498.

[41] Tsai, Wei-Tek, et al. "Rapid Embedded System Testing Using Verification Patterns." IEEE Software 22.4 (2005): 68-75.

[42] U.S. FDA, Design Control Guidance for Medical Device Manufacturers, March 11, 1997. http://www.fda.gov/cdrh/comp/designgd.pdf.

[43] U.S. FDA, General Principles of Software Validation; Final Guidance for Industry and FDA Staff, January 11, 2002. http://www.fda.gov/cdrh/comp/guidance/938.pdf.

[44] Winters, Brian D., and Alan J. Hu. "Source-Level Transformations for Improved Formal Verification." In Proc. of IEEE International Conference on Computer Design (2000): 599.

[45] Zhu, Feng. A Requirement Verification Framework for Real-time Embedded Systems. Diss. Univ. of Minnesota, 2002. Proquest UMI, 2002. 727387361 . 